\title{
N-BODY SIMULATIONS OF ELLIPTICAL GALAXIES
}

\author{
T.S. van Albada \\ Kapteyn Astronomical Institute \\ P.O. Box 800 \\ 9700 AV Groningen \\ The Netherlands
}

\begin{abstract}
N-body simulations are a useful tool for constructing equilibrium models of elliptical galaxies and for the exploration of their kinematical properties, in particular the tumbling rate of the figure about some axis and the internal streaming. As yet little is known about these, except that there is a large variety of possible equilibrium models. It is easy to make triaxial systems that tumble about the short axis, with internal streaming aligned with the rotation axis of the figure. Attempts to construct systems with figure rotation and internal streaming in opposite directions have not been successful. Use of current simulation codes for detailed studies of particle orbits is limited to several (about 10) dynamical times due to non-physical fluctuations in the force field.
\end{abstract}

\section{INTRODUCTION}

Papers presented at this symposium make it clear that our view of the dynamics of elliptical galaxies has changed greatly during the last decade. Much of this is due to the realization that elliptical galaxies are probably triaxial in shape rather than spheroidal. The insignificant rotation of many flattened ellipticals is the main basis for this insight, but a distinct contribution has also been made by $\mathrm{N}$-body simulations. In an early paper on the subject, Binney (1976; see also Aarseth and Binney 1978) showed that violent relaxation from irregular initial conditions does not wipe out all memory of the initial state, and leads to triaxial equilibrium systems. The non-spherical structure is supported by an anisotropic velocity distribution. Later work on dissipationless formation of elliptical galaxies has concentrated on the shape of the radial density profile. Results indicate that violent relaxation starting from cold, irregular initial conditions inescapably leads to a density law that is close to the observed $r^{1 / 4}$ law. For a review see White (1987 - this volume). It is not surprising that $\mathrm{N}$-body simulations are useful for these problems because they probably are the best available tool for exploring the terra incognita of galaxy dynamics. The absence of collisions in simulations of elliptical galaxies is achieved by calculating the force field from a smoothed-out density distribution. Existing codes use either Fourier series or spherical harmonics expansions; see Miller (1978), Wilkinson and James (1982), Villumsen (1982), van Albada (1982), White (1983), 
McGlynn (1984), and Bontekoe (1987).

$\mathrm{N}$-body simulations can also be used to construct equilibrium models. Usually the latter are also made by violent relaxation from non-equilibrium conditions, but less significance is then given to the particular choice of initial conditions. In this way, Miller (1978), Miller and Smith (1979), and Hohl and Zang (1979) constructed tumbling bars from rotating homogeneous spheres. Several aspects of the dynamics of elliptical galaxies, such as orbital structure (Wilkinson and James 1982) and stability (Merritt 1987 - this volume, Stiavelli 1986), can conveniently be studied with N-body simulations. Here, however, a weakness of N-body simulations appears: dynamical principles are not made explicit, e.g. detailed numerical information on orbits is not sufficient to tell why orbits should have a particular shape. Fortunately, more abstract mathematical tools are now available to make up for this deficiency, for example: spectral stellar dynamics (Binney and Spergel 1982), the study of orbits in separable potentials (de Zeeuw 1985), and the orbital density method for constructing equilibrium systems (Schwarzschild 1979).

Somewhat surprisingly, not much work has been done on the exploration of the full range of possible equilibria with $\mathrm{N}$-body simulations. In this paper I will concentrate on work in that area, but first I will discuss briefly some of the limitations of N-body simulations for studies of the dynamics of collisionless systems. (For some comments on N-body methods, see van Albada 1986.)

\section{LIMITATIONS OF COLLISIONLESS N-BODY SIMULATIONS}

All properties of equilibrium models, and of time dependent systems as well, depend on the microscopic structure of the system, that is on the orbits. It is therefore important to look into the quality of N-body orbits, even if one is only interested in global properties. Since orbits in collisionless systems are smooth, numerical procedures can be much simpler than in collisional systems, but the question remains whether present techniques are perhaps too approximate. The fact that the quality of orbits in collisionless $\mathrm{N}$-body systems is hardly ever discussed may indicate that things are not as good as they should be.

As an example, consider the orbits in the equilibrium model discussed by Norman, May and van Albada (1985). This is a nearly prolate equilibrium configuration $(N=20000)$, with $b / a=0.65$ and $c / a=0.64$. Orbits in this model have been classified by monitoring the components of the angular momenta of the individual particles. Nearly all orbits can be classified in this way as belonging to one of the major orbit families (see, however, problem of type switching below). In the above model we find $67 \% \mathrm{X}$-tubes, $6 \% \mathrm{Z}$-tubes and $27 \%$ boxes (including stochastic orbits and misclassified tubes). The large fraction of X-tube orbits reflects the nearly prolate structure of the system. The model is a nicely stationary configuration as far as the global properties are concerned: energy is conserved to within $0.4 \%$ over 25 crossing times and the radius of the $90 \%$ mass shell changes less than $1 \%$ in this period. However, this near constancy of global properties masks considerable changes in the individual particle orbits. Secular changes in the binding energy of individual particles of about $10-20 \%$ occur over a time interval of 50 crossing times. (Similar variations have been observed by Wilkinson, private communication.) These are caused by 'noise' in the force field, due to the limited number of particles, and by truncation errors that are hard to avoid. The presence of noise implies that orbits close to a boundary between families may pass the boundary 


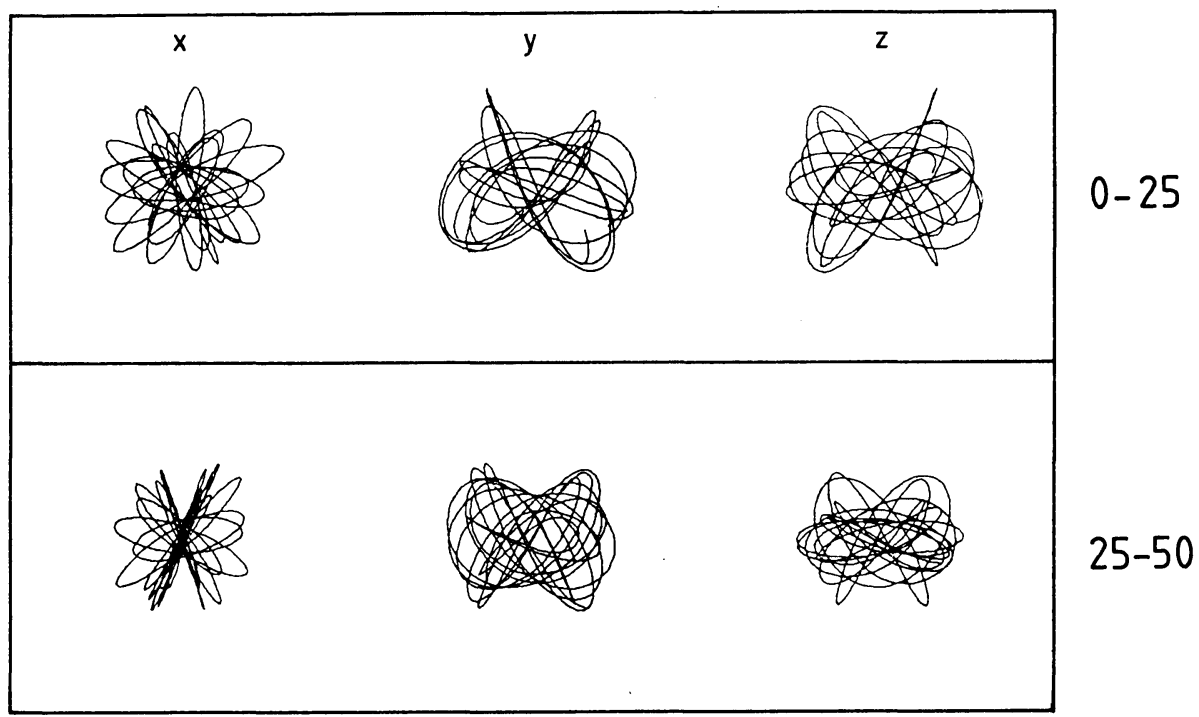

Figure 1. Three projections of an orbit in a triaxial galaxy; from left to right, views along $\mathrm{X}, \mathrm{Y}$, and $\mathrm{Z}$ axes respectively. Upper panel: first 25 time units; the orbit is a long-axis tube. Lower panel: second 25 time units; somewhere in this time interval the orbit switches from a long-axis tube to a box orbit.

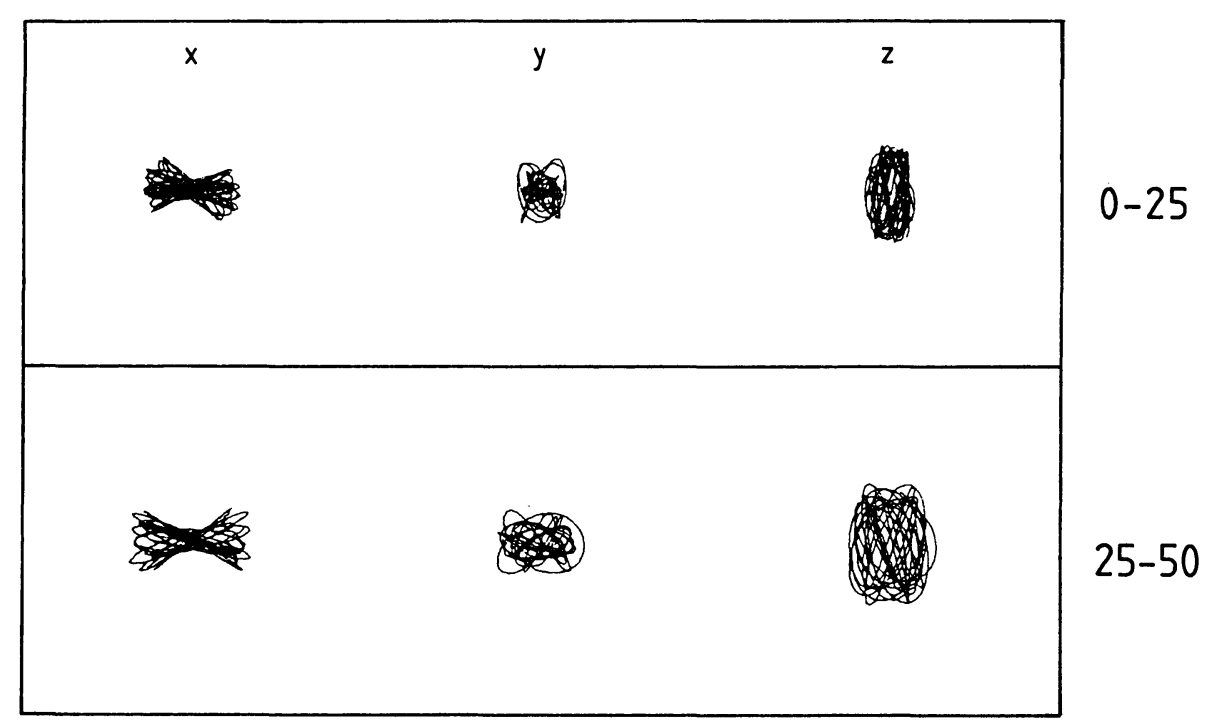

Figure 2. As Figure 1, for box orbit in central region. Note difference in size of orbit for the two time intervals. 
and change family. Thus, the stationary state of a model is an equilibrium in a statistical sense only.

These problems are illustrated in Figures 1 and 2, which show projections of orbits for two time intervals (0-25 and 25-50 crossing times after equilibrium has been reached). The orbit of the particle in Figure 1 is an X-tube during the first 25 crossing times, but somewhere during the second time interval it changes to a box orbit. Note that the 'hole' around the $\mathrm{X}$-axis is rather small so it is relatively easy to pass the center on the 'wrong' side. Most tube orbits have larger angular momenta than the case shown in Figure 1 and for those the problem of type switching does not occur. Figure 2 shows an orbit in the central region. This orbit grows in size as time goes on; thus, the orbital energy is not strictly conserved.

One may conclude that the N-body orbits in collisionless systems are basically correct, even though their shapes and sizes vary somewhat with time. The orbital structure of an equilibrium model should be correct for a period of several (about 10) crossing times, and the study of processes like violent relaxation should be no problem. On the other hand, due to the non-physical variations of the properties of individual particle orbits, it is not yet possible to study evolution caused by slowly changing external force fields, or secular internal evolution.

\section{INTERNAL STREAMING AND TUMBLING}

As demonstrated by Miller (1978), Miller and Smith $(1979,1980)$, Hohl and Zang (1979), and Wilkinson and James (1982), triaxial equilibrium models can be made that tumble about the short axis with constant angular velocity. A large range in tumbling speeds is possible. In addition to tumbling, also called figure rotation, there is internal streaming with respect to the figure in these models. In principle the kinematics of triaxial systems need not be limited to motions about the short axis. By a suitable alignment of the directions of motion of orbits circulating about one of the principal axes (i.e. tube orbits) one can produce internal streaming about an axis that need not coincide with the axis of figure rotation. Both of these axes can, perhaps, have an arbitrary orientation with respect to the principal axes of the system. Many parameters are therefore needed for a complete description of a given configuration. It seems unlikely that all of these can be derived from observation for a given system, even though kinematical models (Binney 1985, Davies and Birkinshaw 1986) show that one can derive interesting constraints.

In this situation $\mathrm{N}$-body simulations can help to restrict the range of possibilities by finding out which configurations can exist as systems in dynamical equilibrium. One way to conduct such experiments is to take a stationary triaxial system in dynamical equilibrium and to induce tumbling by applying an external torque for a limited period of time, allowing the system to relax thereafter. Note that the initial effect of a torque is internal streaming; only when the torque is applied over a sufficiently long time does it induce tumbling. By varying the orientation and strength of the torque one can generate different equilibrium systems. In this way one can determine, for example, whether a triaxial sytem can tumble about its intermediate principal axis or only about the longest or shortest principal axes. (Rotation about the intermediate principal axis is unstable for a solid figure, cf. Goldstein (1980).) Internal streaming about a preferred direction can also be introduced by monitoring the sense of circulation of the particles about the principal axes and by reversing the direction of motion of some of the particles which 


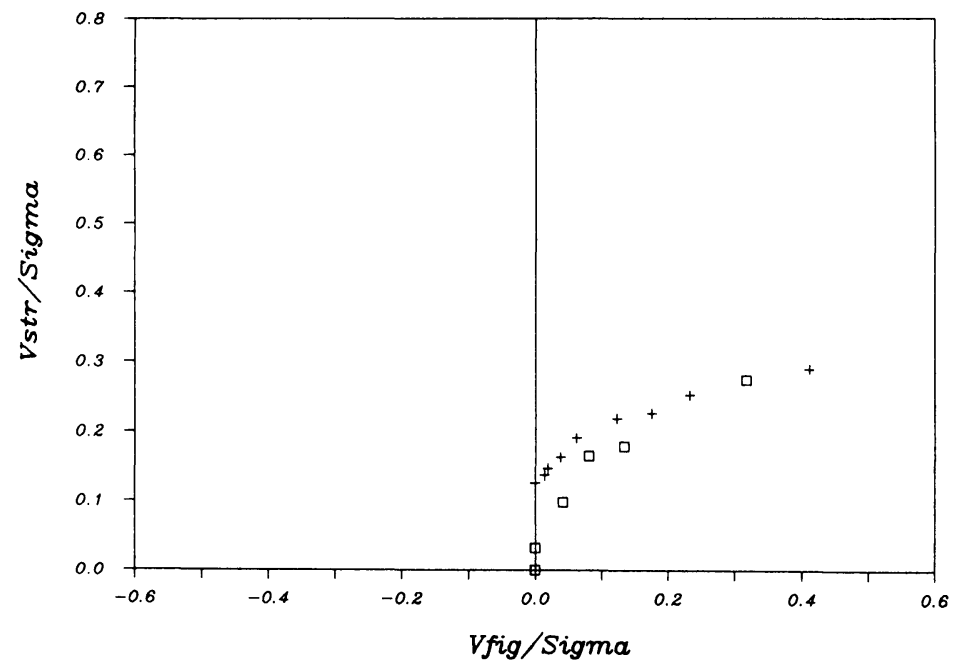

Figure 3. Relation between tumbling and streaming velocities for two sequences of triaxial models discussed in text.

circulate in the 'wrong' sense.

Experiments of this type were carried out by the author for a nearly prolate equilibrium model, similar to the one described in section 2 . Only the simple case that both figure rotation and internal streaming are about the short axis was considered. Thus, the final models are tumbling bars. In this sense our models are similar to those of Miller and Smith (1980), who have already emphasized the large variety of possible equilibrium models. Two sequences of tumbling models were made. For one sequence the starting model had no internal streaming (open squares in Figures 3 and 4). For the other sequence the maximum amount of internal streaming was introduced by aligning the senses of circulation of all Z-tube orbits in the initial configuration (crosses in Figures 3 and 4). However, because the fraction of $\mathrm{Z}$-tube orbits in the model is small the net streaming velocity in the starting model is limited. For both sequences application of the external torque not only introduces tumbling about the $\mathrm{Z}$-axis but also causes additional internal streaming.

In Figure 3 the relation between tumbling and streaming in the final models is represented in terms of $v_{f i g} / \sigma_{0}$ and $v_{s t r} / \sigma_{0}$, where $v_{f i g}$ and $v_{s t r}$ are respectively the figure rotation and streaming velocity at the half-mass radius (as seen by an observer in the XY-plane) and $\sigma_{0}$ is the central velocity dispersion in one dimension. Each symbol in this Figure represents one possible equilibrium model. The main conclusions can be summarized as follows:

(i) Stationary, i.e. non-tumbling, systems with internal streaming are possible equilibrium configurations (models indicated by cross and square on vertical axis). This may seem a trivial result, but it is nice to see that such models are stable.

(ii) All tumbling systems in Figure 3 posses internal streaming. It is not clear whether this result is a general property of triaxial systems. It could perhaps be a result of the special way in which tumbling was introduced in these models, namely 


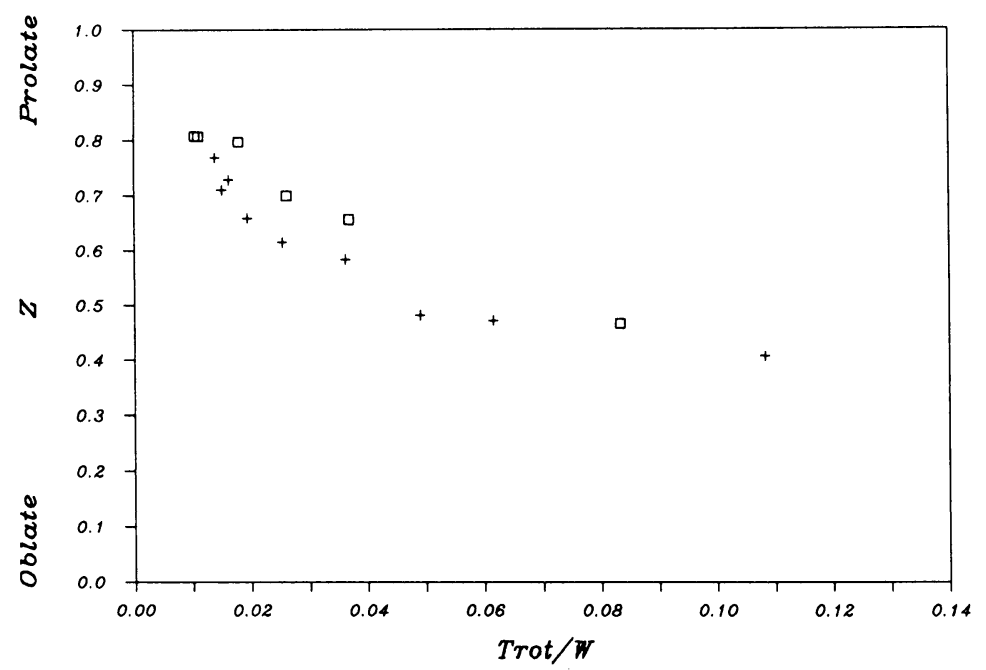

Figure 4. Shape versus amount of rotational kinetic energy, $T_{\text {rot }} / W$, for triaxial models discussed in text. Shape is measured by the triaxiality parameter $Z=$ $(1-b / a) /(1-c / a)$. Note increasing oblateness with increasing $T_{\text {rot }} / W$.

through the application of an external torque.

(iii) Tumbling in a direction opposite to the internal streaming does not appear possible. This is evident from the absence of models in the left part of Figure 3. Attempts were made to make such models by applying a torque in a direction opposite to the internal streaming, but without success. What happens in such experiments is that the torque reduces the internal streaming; it does not induce tumbling as long as internal streaming is opposed to the torque. This behaviour is quite different from that when torque and internal streaming are parallel. Then the system responds promptly by increasing its tumbling rate. A similar conclusion on the alignment of figure rotation and internal streaming has been reached by Vietri (1986), who tried, without success, to make a specific triaxial model with figure rotation opposed to internal streaming using Schwarzschild's orbital-density method.

Some other features of these models are worth noting. First, a large range in tumbling speeds about the short axis is possible. For the models in Figure 3 the tumbling periods range from $4.2 \times 10^{8}$ to $75 \times 10^{8}$ yrs (adopting a total mass of $2 \times 10^{11} M_{\odot}$ and a half-mass radius of $4 \mathrm{kpc}$ ). Second, the models in Figure 3 vary in shape (see Figure 4); systems become increasingly oblate if the strength of the torque is increased. Finally, there is a tight correlation between the amount of figure rotation and internal streaming for the models in Figure 3. In a general sense this agrees with earlier results from $\mathrm{N}$-body experiments: rapidly rotating systems develop a rapidly tumbling bar (Hohl and Zang 1979, Miller and Smith 1979), while slowly tumbling triaxial systems have a modest amount of internal streaming (Wilkinson and James 1982). On the other hand, the tightness of the correlation in Figure 3 is no doubt a consequence of the use of a single (nearly prolate) starting configuration, with at most a small amount of internal streaming. 
More oblate models have a larger fraction of short-axis tube orbits (Statler 1986), and by aligning the directions of motion of these orbits one can probably make a non-tumbling system with a fairly large amount of internal streaming.

The above discussion shows that $\mathrm{N}$-body simulations form a versatile tool to study the range in stable equilibria of elliptical galaxies, but present work still has the character of an initial exploration. One should keep in mind that real elliptical galaxies form a subset of the stable $\mathrm{N}$-body systems due to additional constraints acting during the formation phase; the physics of galaxy formation determines the details of the collapse and subsequent violent relaxation phase, and thereby specifies the resulting equilibrium system.

I am indebted to Andrew May for carrying out most of the analysis presented in section 2 and to Linda Sparke for comments.

\section{REFERENCES}

Aarseth, S.J. and Binney, J.J., 1978. Mon. Not. Roy. Astr. Soc., 185, 227.

Binney, J., 1976. Mon. Not. Roy. Astr. Soc., 177, 19.

Binney, J., 1985. Mon. Not. Roy. Astr. Soc., 212, 767.

Binney, J. and Spergel, D., 1982. Astrophys. J., 252, 308.

Bontekoe, T.R., 1987. Ph.D. Thesis Groningen University, in preparation.

Davies, R.L. and Birkinshaw, M., 1986. Astrophys. J., 303, L45.

de Zeeuw, T., 1985. Mon. Not. Roy. Astr. Soc., 216, 273.

Goldstein, H., 1980. Classical Mechanics, $2^{\text {nd }}$ ed., p. 210, Addison-Wesley.

Hohl, F., and Zang, T.A., 1979. Astron. J., 84, 585.

McGlynn, T.A., 1984. Astrophys. J., 281, 13.

Merritt, D., 1987. This volume, p. 315.

Miller, R.H., 1978. Astrophys. J., 223, 122.

Miller, R.H. and Smith, B.F., 1979. Astrophys. J., 227, 407.

Miller, R.H. and Smith, B.F., 1980. Astrophys. J., 235, 793.

Norman C.A., May, A. and van Albada, T.S., 1985. Astrophys. J., 296, 20.

Schwarzschild, M., 1979. Astrophys. J., 232, 236.

Statler, T.S., 1986. Ph.D. Thesis Princeton University.

Stiavelli, M., 1986. Ph.D. Thesis Scuola Normale Superiore, Pisa.

van Albada, T.S., 1982. Mon. Not. Roy. Astr. Soc., 201, 939.

van Albada, T.S., 1986. In: The Use of Supercomputers in Stellar Dynamics, eds.

P. Hut and S. McMillan, Springer Verlag. p. 23.

Vietri, M., 1986. Astrophys. J., 306, 48.

Villumsen, J.V., 1982. Mon. Not. Roy. Astr. Soc., 199, 171.

White, S.D.M., 1983. Astrophys. J., 274, 53.

White, S.D.M., 1987. This volume, p. 339.

Wilkinson, A. and James, R.A., 1982. Mon. Not. Roy. Astr. Soc., 199, 171. 


\section{DISCUSSION}

Quinn: If streaming around a figure were present and comparable to the orbital velocities of stars in the galaxy, then when the galaxy appears to be nearly round on the sky we would expect to see a large $V / \sigma$ for a small ellipticity $\epsilon$. There appear to be very few large $V / \sigma$ small $\epsilon$ galaxies among observed ellipticals. This may then constrain the contribution of streaming velocities to $V$.

van Albada: If I understand correctly, the situation you describe is that of a nearly prolate galaxy, with internal streaming about the short axis, which is seen end-on. Such an orientation has a rather low probability. One should probably take a better look at the statistics to see whether the observations give useful constraints.

Vietri: (i) I do not understand why you cannot set up counter-streaming motions for sufficiently low values of $\Omega_{p}$. Suppose in fact $\Omega_{p}=0$. Then you can get stars streaming in either direction. Now give a gentle tug to the figure. I would expect the model to show some degree of counterstreaming. Can you say why you do not find such solutions? (ii) Gravitational torques work more subtly than one would think naively. Tremaine and Weinberg (1984, Mon. Not. R. astr. Soc., 209, 729) showed that the average star is not spun up at any given time. Only orbits momentarily in resonance are spun up. This is different from what you do, since you spin up all orbits, at the same time. Can you say whether your results would be effected by including this effect?

van Albada: Perhaps the $\mathrm{N}$-body simulations are too rough. Tumbling can only be measured when it exceeds about one revolution per $10^{10}$ years. As regards your second point, the temporal behavior of the force seen by a star in the torque experiments is indeed quite different from that caused by a satellite in circular orbit. I don' $t$ know what would happen in the latter case.

Bishop: For the $\mathrm{N}$-body simulation, how do you specify the initial conditions and how well does the distribution function retain its initial form?

van Albada: The equilibrium model I showed was generated from non-equilibrium initial conditions, as described in Norman, May and van Albada (1985). Once equilibrium has been reached, the shape of the distribution of individual particle energies is well conserved.

Lake: Freeman's models (1966, Mon. Not. $R$. astr. Soc., 133, 47; 134, 1, 15) counterstream. They certainly aren't stable, but one could try them as an initial state.

van Albada: Yes, that could be tried.

White: I found your pictures of the long term evolution of orbits to be very instructive but I think your conclusions may be too pessimistic. It is important to bear in mind that present $\mathrm{N}$-body techniques were not set up primarily to attack problems of this kind and we may be able to do significantly better by some careful attention to the algorithms. 
van Albada: I agree that that should be done.

Schwarzschild: The last speaker is, I feel, too terribly modest regarding the accuracy and completeness he has reached. From his results as they stand one can, I think, draw some quite secure general conclusions. Specifically I would conclude from his results-bolstered by some recent work of Mario Vietri-that if one observes an elliptical to rotate with a substantial velocity, say $V / \sigma>0.2$, then the figure rotation, if any, must be in the same sense as the observed net streaming, i.e. no strong counterstreaming.

Spergel: While $\mathrm{N}$-body techniques may be robust when there are not many resonant orbits, resonant orbits might introduce spurious stochasticity. This is not a problem for loop orbits. However, when there are many resonant tori, the fluctuations in the $\mathrm{N}$-body code might scatter the orbits from one torus to a nearby torus. The Liapunov coefficients for particles with finite separation might be quite large. Energy conservation is not a good diagnostic of this problem, since the neighboring tori may have similar energies but visit very different regions of phase space.

van Albada: The point I wanted to make is that even the necessary condition that individual particle energies are conserved, is not fulfilled in present $\mathrm{N}$-body simulations. I agree that this condition would not be a sufficient one for strict equilibrium.

Statler: I hope that Dave Spergel's comment does not discourage people from looking at the stellar orbits in $\mathrm{N}$-body models. We can use Schwarzschild's method and linear programming to find what kind of self-consistent solutions can exist, but we need the $\mathrm{N}$-body models to tell us which of them can actually be made. So I would like to encourage the $\mathrm{N}$-body experts, especially those who simulate mergers, to do what Althea Wilkinson has done, and calculate distribution functions for their models, or at least find the mass fractions in the major orbit families.

Petrou: From the computation of orbits in two-dimensional potentials I have done, I know that in order to study high order resonances and their effect you need at least 7-8 significant figure accuracy in the energy conservation. With a $0.4 \%$ accuracy in the energy you can only see the effect of the very simple resonances.

Gerhard: (i) Can I interpret your results to the effect that the observed rotation velocity in an edge-on bulge at given radius $R$ and highest slit position $z$, gives an upper limit to the figure rotation velocity at $R$, and hence a lower limit to the corotation radius, if this bulge were triaxial? In this case, corotation in NGC 4565 would be at least at $\sim 15 \mathrm{kpc}$ (using data from Kormendy and Illingworth 1982, Astrophys. J., 265, 637). (ii) Concerning the role of resonant orbits: Is the orbit sample you showed representative of all orbits in this system, and do you have a surface of section for the potential of this model to see whether resonant orbits are important in its phase-space and/or preferentially populated?

van Albada: (i) Rejecting the possibility of streaming and tumbling in opposite directions, I would indeed interpret any observed rotation as an upper limit to the figure rotation. (ii) I mainly showed orbits with "problems," leaving out wellbehaved tube orbits. No further analysis of the orbits has been made. 


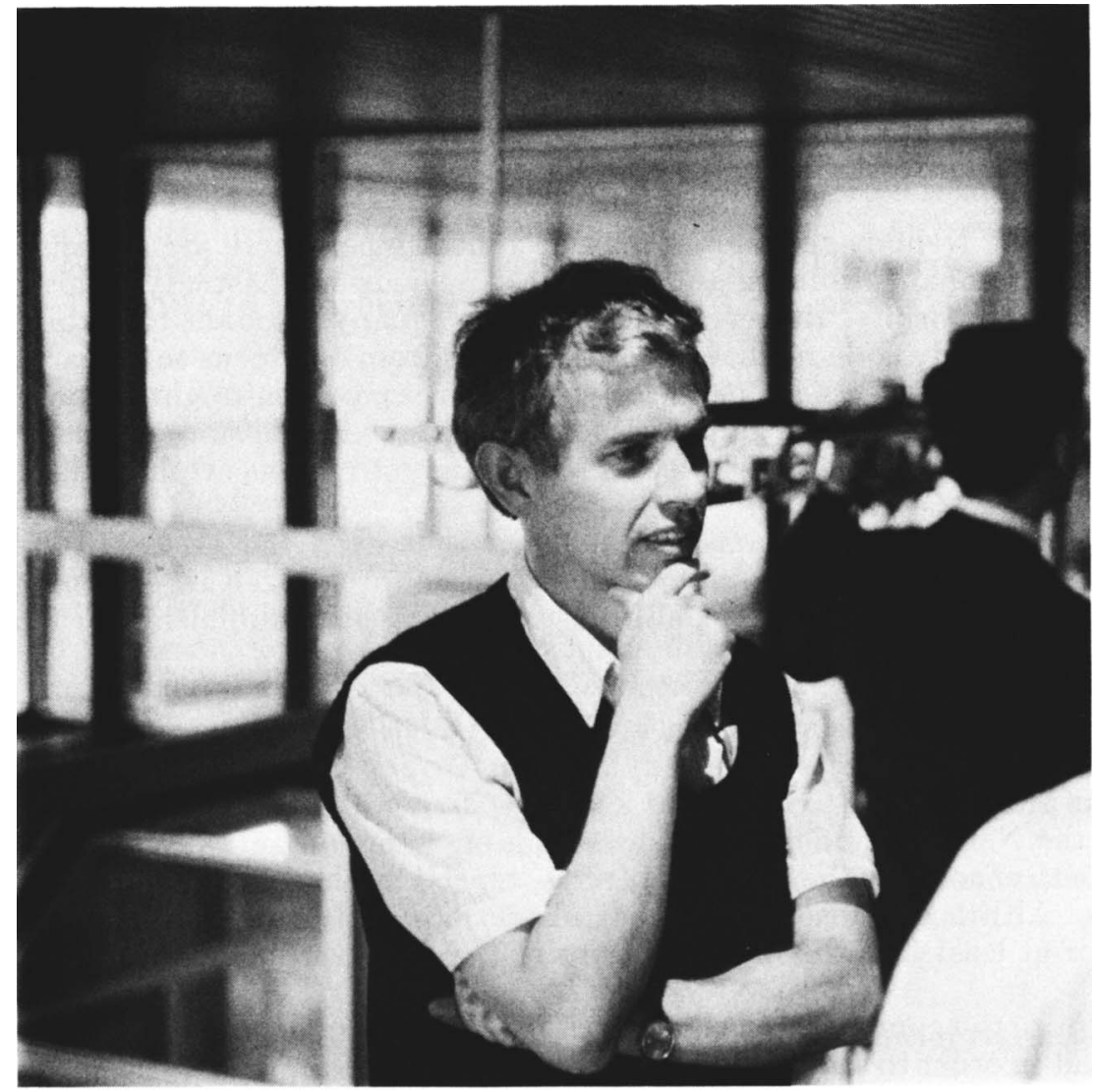

Tjeerd van Albada. 